\title{
New multiobjective optimization algorithm using NBI-SASP approaches for mechanical structural problems
}

\author{
Samira El Moumen ${ }^{1, *}$ (D) and Siham Ouhimmou ${ }^{2}$ \\ ${ }^{1}$ LIMSAD, Department of Mathematics and computing, Hassan 2 University, Ain Chock Sciences Faculty, Km8 Route \\ El Jadida, BOP 5366 Maarif, Casablanca, Morocco \\ ${ }^{2}$ LM, Department of Physics, Hassan 2 University, Ain Chock Sciences Faculty, Km8 Route El Jadida, BOP 5366 Maarif, \\ Casablanca, Morocco
}

Received: 26 January 2021 / Accepted: 7 November 2021

\begin{abstract}
Various engineering design problems are formulated as constrained multi-objective optimization problems. One of the relevant and popular methods that deals with these problems is the weighted method. However, the major inconvenience with its application is that it does not yield a well distributed set. In this study, the use of the Normal Boundary Intersection approach (NBI) is proposed, which is effective in obtaining an evenly distributed set of points in the Pareto set. Given an evenly distributed set of weights, it can be strictly shown that this approach is absolutely independent of the relative scales of the functions. Moreover, in order to ensure the convergence to the Global Pareto frontier, NBI approach has to be aligned with a global optimization method. Thus, the following paper suggests NBI-Simulated Annealing Simultaneous Perturbation method (NBI-SASP) as a new method for multiobjective optimization problems. The study shall test also the applicability of the NBI-SASP approach using different engineering multi-objective optimization problems and the findings shall be compared to a method of reference (NSGA). Results clearly demonstrate that the suggested method is more efficient when it comes to search ability and it provides a well distributed global Pareto Front.
\end{abstract}

Keywords: Global optimization / hybrid method / simultaneous perturbation / simulated annealing / multiobjective optimization / normal boundary intersection approach / pareto front

\section{Introduction}

Optimization in engineering has become a vital component with the growth of the capabilities of computers nowadays. In today's time, complicated calculations can be done in a very short time compared to years ago. Consequently, the use of numerical optimization has witnessed a dramatic increase. A significant amount of designing is usually performed intuitively. However, technical analyses involving numerical optimization can contribute a great deal to the amelioration of the designs see [1-4].

What characterizes any problem of engineering design is the presence of various objectives $[5,6]$. When all these objectives are taken into account, their conflicting nature finds it challenging to find an optimal solution to the problem. A set of solutions in which improving one objective can deteriorate the other ones is referred to as the Pareto set of non-dominated solutions and approximating it contributes to understanding it in depth as well as

\footnotetext{
* e-mail: selmoumen@yahoo.fr
}

assisting the process of decision-making. Two main methods for searching for the Pareto Front have been introduced in the last two decades: weighted method $[7,8]$ and $\varepsilon$-constraint method [7,8], Ehrgott and Gandibleux (2002), [9-12]. The issue with the Weighted method is that it does not have the ability to solve non-convex objective space and it does not yield a well-distributed set. On the other hand, the $\varepsilon$-constraint method deals with nonconvex space, but still does not yield a well-distributed set as well. This paper purports to propose the use of the Normal Boundary Intersection approach (NBI) [13] that allows us to handle non-convex objective space and yields a well distributed set. In order to establish a transition to the global Pareto frontier, this paper is set to combine the NBI approach with a proposed global optimization method entitled Simulated Annealing Simultaneous Perturbation method (SASP). This method involves hybridization of the Simulated Annealing method and a moderated method where the gradient is estimated by using the simultaneous perturbation. This paper endeavors to suggest NBISimulated Annealing Simultaneous Perturbation method (NBI-SASP) to ameliorate the efficiency of 
the optimization algorithms. Furthermore, despite the achievement of some improvements with regards to proposed algorithms of multi-objective design optimization, the complicated nature of design problems with conflicting objectives, wide solution space, and premature convergence to local optimum is considered as a drawback for the solution and computational cost of multi-objective design optimization problems. In order to compare the results, NSGA-II shall be used as a method of references $[6,8]$. Then, a simulation of the results on various difficult test problems shall reveal that NBI-SASP outperforms NSGA-II with regards to detecting a diverse set of solutions and converging near the true Paretooptimal set.

\section{Multi-objective optimization problem}

A multi-objective optimization problem is defined as follows:

$$
\begin{aligned}
& \min F(x)=\left(f_{1}(x), f_{2}(x), \ldots, f_{k}(x)\right)^{T} \\
& \text { s.t. } x=\left(x_{1}, x_{2}, \ldots, x_{n}\right)^{T} \in S
\end{aligned}
$$

where $f_{1}(x), f_{2}(x), \ldots, f_{k}(x)$ are the $k$ objectives functions, $x_{1}$, $x_{2}, \ldots, x_{n}$ are the $\mathrm{n}$ optimization parameters, and $S \in \mathbb{R}^{n}$ is the solution or parameter space.

$f_{1}^{*}, f_{2}^{*}, \ldots, f_{k}^{*}$ will be used to denote the individual minima of each respective objective function, and the utopian solution is defined as $F^{*}=\left(f_{1}^{*}, f_{2}^{*}, \ldots, f_{k}^{*}\right)$. As $F^{*}$ simultaneously minimizes all objectives, it is an ideal solution that is rarely feasible. Figure 1 provides a visualization of the nomenclature.

Note that because $F(x)$ is a vector, if the components of $F(x)$ are involved in a competition, no unique solution can be found to this problem. Nevertheless, the concept of noninferiority (also called Pareto optimality) is necessary to characterize the objectives. A solution where improving one objective results in the deterioration of another is called a non-inferiority solution. Obviously, a final design solution is preferred to be a member of the Pareto optimal set. Therefore, Pareto optimal solutions are also called nondominated or efficient solutions. Once a final solution is chosen from the set of Pareto optimal solutions, no other existing solution shall be better in all attributes. However, in case the solution does not belong to the Pareto optimal set, improvements can be made without deteriorating any objective, and, therefore, it is not a rational choice.

\section{The NBI-SASP method}

\subsection{Normal boundary intersection (NBI)}

Normal Boundary Intersection is a method developed in 1998 by Das and Dennis. It aims to identify the Pareto front for a multi-objective optimization problem, it is proven that this method has succeeded in producing a uniform set of Pareto front points, and this gives NBI an advantage over the other methods used before, weighting method and $\varepsilon$-constrain method.

Let $x_{i}^{*}$ and $f_{i}^{*}$ denote respectively the minimizer and minimum value of the $f_{i}$ and let $F^{*}$ denote the shadow

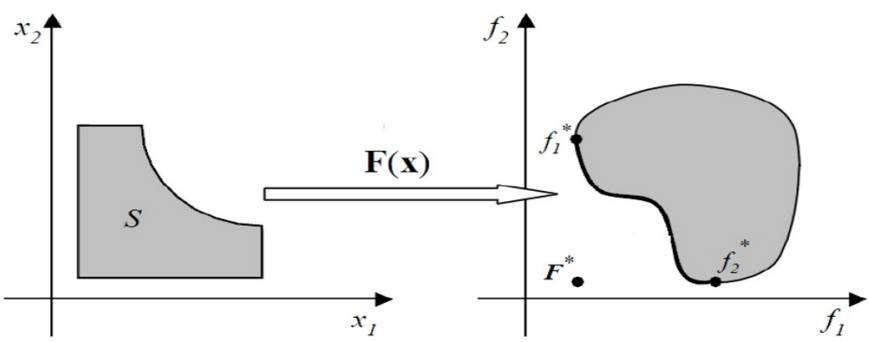

Fig. 1. Two dimensional problem with two objectives.

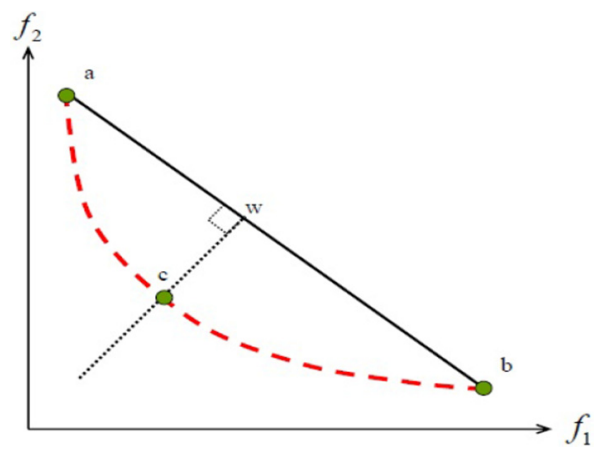

Fig. 2. An illustrative integrated design.

minimum, i.e., the vector whose components are $f_{i}^{*}$. Consider the shifted pay-off matrix $\Phi$ whose $i^{t} h$ column is $F\left(x_{i}^{*}\right)-F^{*}$. The Convex Hull of Individual Minima or CHIM is defined as the set of points that are convex combinations of the columns of $\Phi$, i.e., $\left\{\Phi \beta: \beta_{i} \geq 0\right.$, $\left.\sum_{i} \beta_{i}=1\right\}$.

For a two dimensional problem illustrated in Figure 2, CHIM is represented by segment $\mathrm{AB}$.

The main purpose of NBI is to select an even spread of points on the CHIM (for example $\mathrm{W}$ in 2), and find the intersection point between the efficient frontier and a set of parallel normals resulting from the chosen set of points on the CHIM ( $\mathrm{C}$ in 2). Taking into consideration a convex combination parameter vector $\beta$, and a normal direction $\mathrm{n}$ that points towards the origin, the point of intersection between the normal emanating from $\Phi \beta$ and the efficient frontier can be found by solving the following $N B I_{\beta}$ subproblem:

$$
\left\{\begin{array}{l}
\text { Maximize } t \\
\text { Subject to }: x \in A \\
\Phi \beta+t n=F(x)-F^{*}
\end{array}\right.
$$

where $A$ is the set of feasible solution.

A solution to the sub-problem $N B I_{\beta}$ for different settings of $\beta$ can generate various points on the efficient frontier. The $\beta$ parameter's advantage is that an even spread of $\beta$ parameters is related to an even spread of points on the CHIM. Thus, in order to ensure the convergence to the global Pareto frontier, a marriage of the NBI approach with a global optimization method is inevitable Figure 3. 


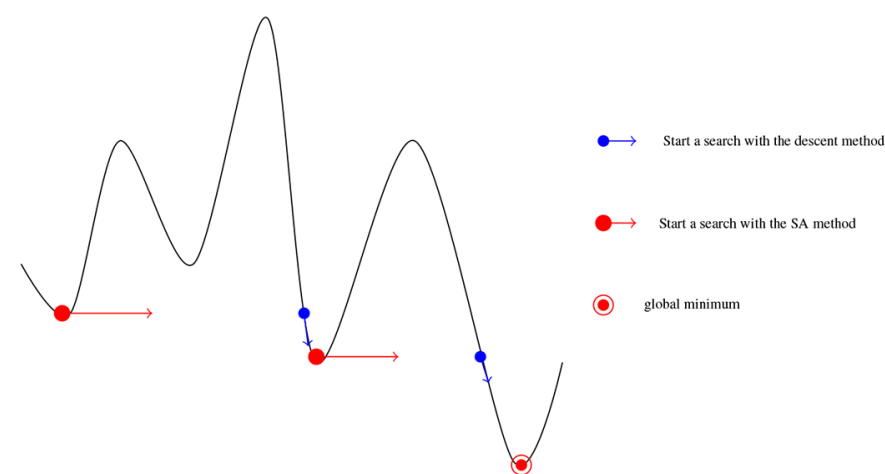

Fig. 3. The SASP organizational chart.

\subsection{The global optimization method (SASP)}

The SASP method offers a good compromise between exploration and exploitation, it is based on two main ideas, the first idea comes from the fact that the simulated annealing algorithm e.g. [14-18], Kirkpatrick et al. (1983), [18] can easily escape from a local optimum, and the second idea is that gradient descent methods converge rapidly towards the local minimum.

This method presents two phases that are executed alternately. In the first phase a local search is run using a descent method where the gradient is estimated by using the simultaneous perturbation [19], from a starting point $x_{0}^{i}$. It will converge (presumably) to a minimum (local) $x_{i}^{*}$. And to deviate from this local solution, the second phase runs using some iterations of the simulated annealing algorithm to find the next best point. Then we take this point $x_{0}^{i+1}$ as a starting point and start a new search with the gradient method. This procedure continues until convergence and finally we find the overall solution [15].

\subsection{The NBI-SASP algorithm}

Flow chart of the NBI algorithm for generating Pareto optimal sets is briefly described in the following Figure 4.

\section{Numerical example}

In this section, we shall introduce a solution for three problems in structural mechanics [20-26]. The application of these solutions will be done using both methods. First using the NBI-SASP approach, and then a close comparison to the NSGA reference approach will be made to check which one will have more efficiency.

\subsection{The two bar truss design problem}

As can be seen in Figure 5, the truss carries a specific load without elastic failure. Therefore, the design objectives involve first and primarily reducing the volume of the structure (which equals less fabrication costs). Secondly, the next step is to reduce or minimize the stresses in each of

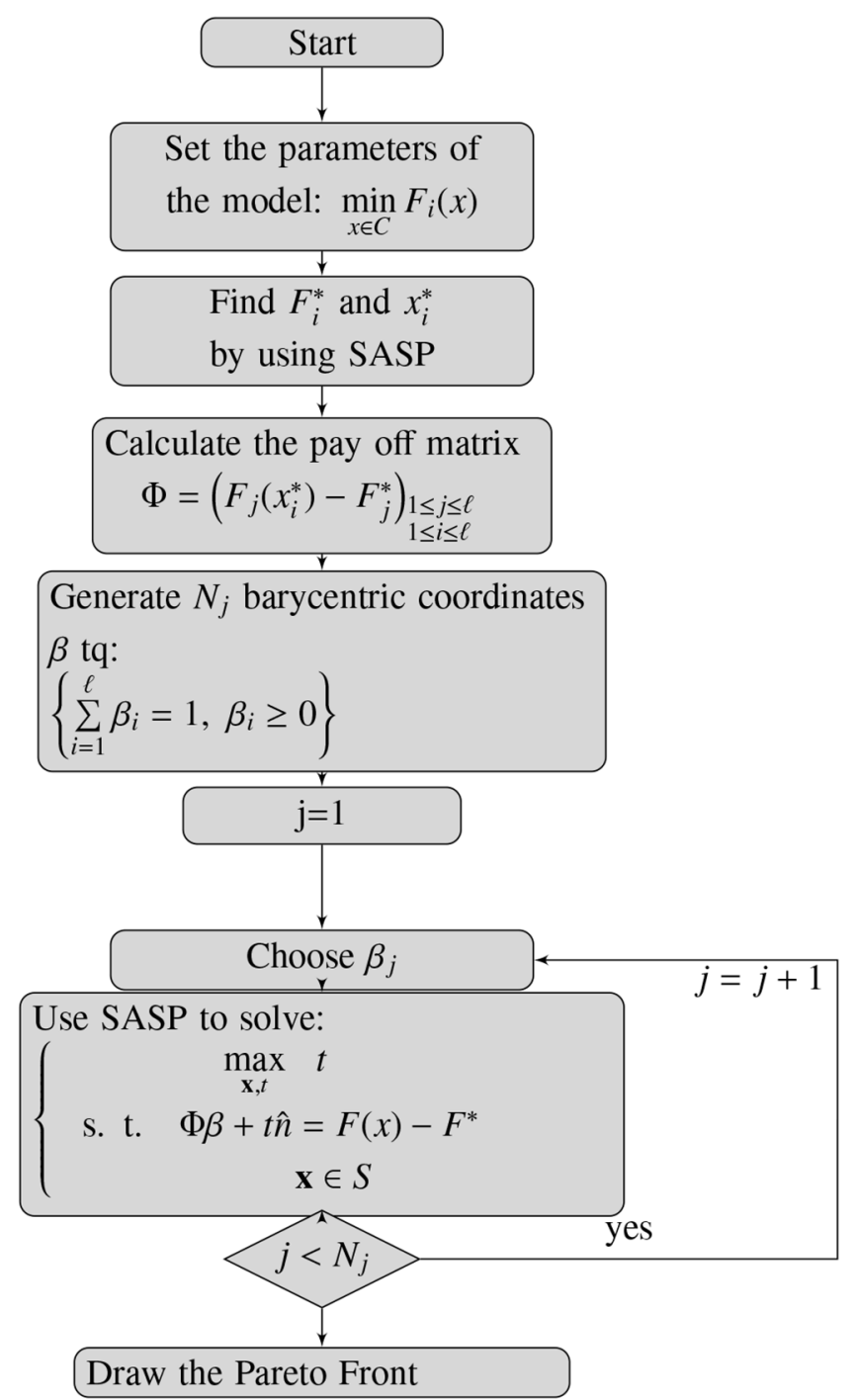

Fig. 4. Flow chart of the NBI algorithm for generating Pareto optimal sets.

the two members AC and BC. For this reason, any design problem involves a two-objective optimization problem for three variables: vertical distance $\mathrm{y}$ between $\mathrm{B}$ and $\mathrm{C}$ (metres), length $x_{1}$ of $\mathrm{AC}$ (metres), and length $x_{2}$ of $\mathrm{BC}$ in (metres).

And the mathematical description of the problem is as follows

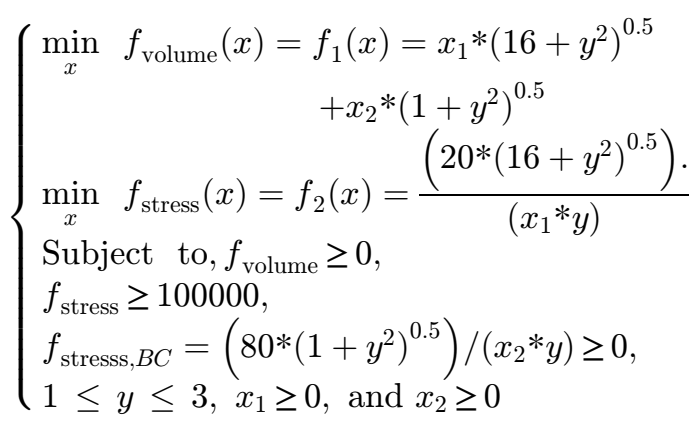




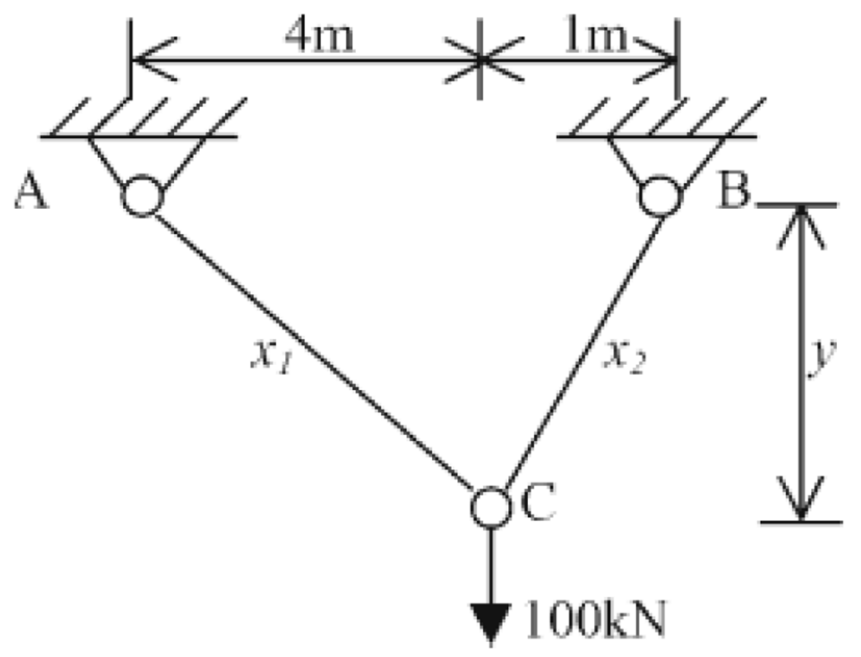

Fig. 5. The two-bar truss design problem.

\subsection{The I-Beam design problem}

The purpose of multi-objective optimization for the I Beam design (see Fig. 6), is to find the optimal compromise between the dimensions of the concrete I-Beam and geometric and strength constraints [27-29]. The two objective functions are simultaneous minimization of $f_{1}$ cross sectional area of the I-Beam, and $f_{2}$ the static deflection of the I-Beam taking into consideration the orthogonal and cross sectional forces $P=600 \mathrm{KN}$ and $Q=50 k N$.

The problem definition is written below

$$
\left\{\begin{array}{l}
\min _{x} f_{\text {Area }}(x)=f_{1}(x)=2 * x_{2} * x_{4}+x_{3}^{*}\left(x_{1}-2 * x_{4}\right) \\
\min _{x} f_{\text {Displacement }}(x)=f_{2}(x)=\frac{\left(P^{*} L^{2}\right)}{\left(48^{*} E^{*} I\right)} \\
\text { Subject to, } g(x)=\frac{M y}{Z 1}+\frac{M z}{Z 1}-\sigma_{1} \geq 0 \\
\quad 10 \leq x_{1} \leq 80,10 \leq x_{2} \leq 50,0.9 \leq x_{3} \leq 5 \\
\quad 0.9 \leq x_{4} \leq 5
\end{array}\right.
$$

where

$$
\begin{gathered}
I=\frac{1}{12}\left(x_{3}\left(x_{1}-2 x_{4}\right)^{3}+2 x_{2} x_{4}\left(4 x_{4}^{2}+3 x_{1}\left(x_{1}-2 x_{4}\right)\right)\right) \\
E=2.10^{4} K N / \mathrm{cm}^{2}, \sigma_{a}=16 K N / \mathrm{cm}^{2} \\
P=600 K N, \text { and } Q=50 K N \\
M_{y}=\frac{P * L}{4}, \text { and } M_{z}=\frac{Q * L}{4} \\
Z_{y}=\frac{1}{6 x_{1}}\left(x_{3}\left(x_{1}-x_{4}\right)^{3}+2 x_{2} x_{4}\left(4 x_{4}^{2}+3 x_{1}\left(x_{1}-2 x_{4}\right)\right)\right)
\end{gathered}
$$

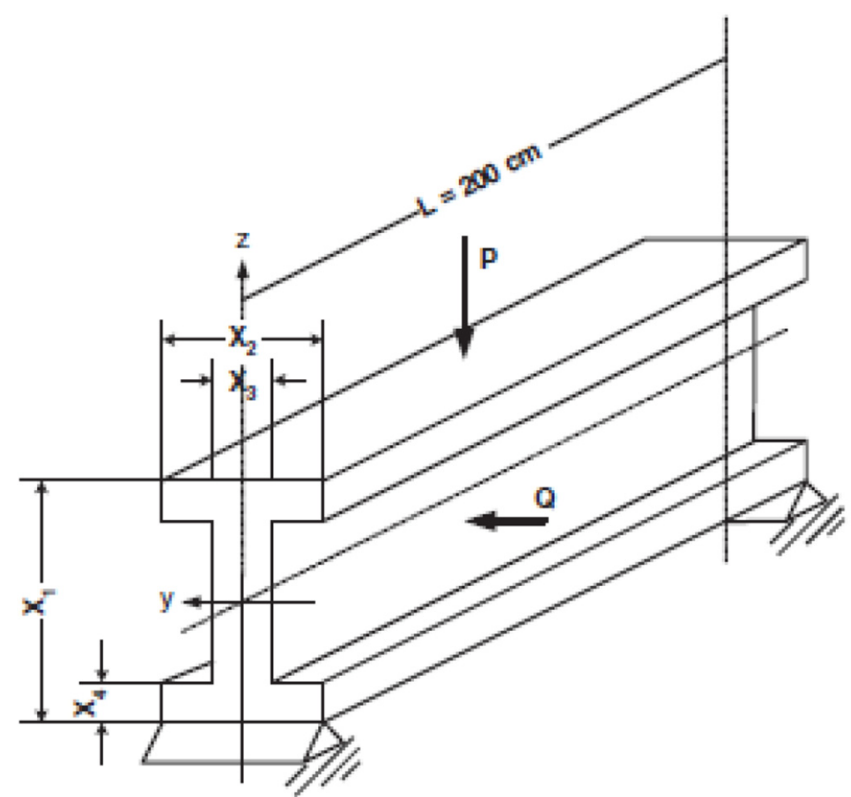

Fig. 6. The I-Beam design example.

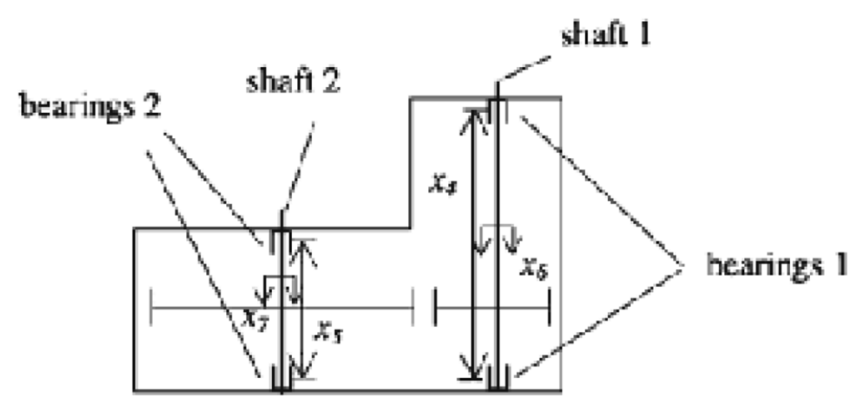

Fig. 7. The disc brake design optimization problem.

$$
Z_{z}=\frac{1}{6 x_{2}}\left(\left(x_{1}-x_{4}\right) x_{3}^{3}+2 x_{4} x_{2}^{3}\right) .
$$

\subsection{The disc brake design problem}

One of the pertinent structural multi-objective optimization problems is the optimization problem of the disk brake design. It is mentioned here to exemplify the effects of the algorithm NBI-SASP compared to other algorithms without improvements for solving multi-objective design optimization problems such as NSGA-II.

The design has as an objective to minimize the mass of the brake as well as the stopping time [5,30,31] The four variables are defined for the disc brake mathematical optimization model as $x_{1}$, inner radius of the discs; $x_{2}$, outer radius of the discs; $x_{3}$, engaging force; and $x_{4}$, number of the friction surface Figure 7 . The objective functions and constraints of the disc brake design optimization model are: 


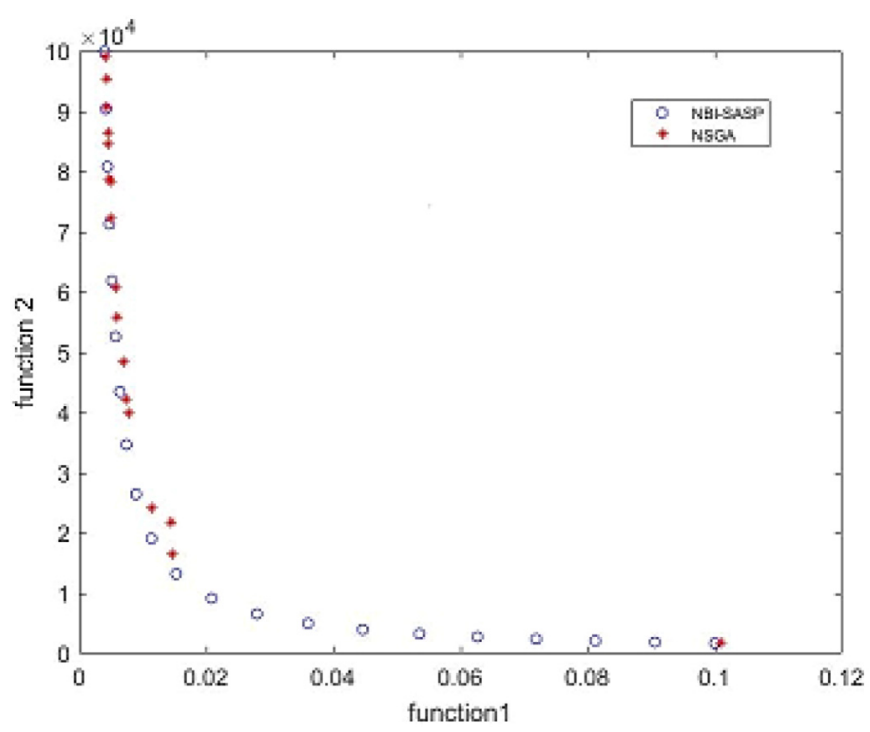

Fig. 8. The solutions obtained by NBI-SASP and NSGA-II on two-bar truss design problem.

$$
\begin{aligned}
& \left(\min _{x} f_{\text {Mass }}(x)=f_{1}(x)=4.9 * 10^{-5 *}\left(x_{2}^{2}-x_{1}^{2}\right) *\left(x_{4}-1\right),\right. \\
& \min _{x} f_{\text {Time }}(x)=f_{2}(x)=\frac{9.82 * 10^{6 *}\left(x_{2}^{2}-x_{1}^{2}\right)}{x_{3} * x_{4}\left(x_{2}^{3}-x_{1}^{3}\right)} \text {, } \\
& \text { Subject to, } g_{1}(x)=\left(x_{2}-x_{1}\right)-20 \geq 0 \text {, } \\
& \left\{\begin{array}{l}
g_{2}(x)=30-2.5 *\left(x_{4}+1\right) \geq 0, \\
g_{3}(x)=0.4-\frac{x_{3}}{3.14^{*}\left(x_{2}^{2}-x_{1}^{2}\right)} \geq 0,
\end{array}\right. \\
& g_{4}(x)=1-\frac{2.22^{*} 10^{-3 *} x_{3} *\left(x_{2}^{3}-x_{1}^{3}\right)}{\left(x_{2}^{2}-x_{1}^{2}\right)^{2}} \geq 0, \\
& g_{5}(x)=\frac{2.66 * 10^{-2} * x_{3} * x_{4} *\left(x_{2}^{3}-x_{1}^{3}\right)}{\left(x_{2}^{2}-x_{1}^{2}\right)}, \\
& 55 \leq x_{1} \leq 80,75 \leq x_{2} \leq 110,1000 \leq x_{3} \leq 3000, \\
& 2 \leq x_{4} \leq 20 \text {. }
\end{aligned}
$$

\section{Results}

In the tree problem, the nonlinear constraints can cause difficulties in finding the Pareto solutions. As shown in Figures 8-10 it is clearly that the NBI, based on the hybrid method, have exclude the non-Pareto and local Pareto points, compared with the result obtained by the NSGA-II algorithm. Also all the figure can be shown that our approach guarantees uniform distribution of the Pareto solutions.

Taking into account the whole analysis, it can be deduced that the NBI SPSA solutions are better then NSGA-II with regards to both the closeness to the true optimum and their spread for all test problems employed in the study. The NBI SPSA does not face any challenges in order to reach an effective spread of Pareto-optimal solutions for constrained multi-objective optimization. The results yielded for engineering design problems

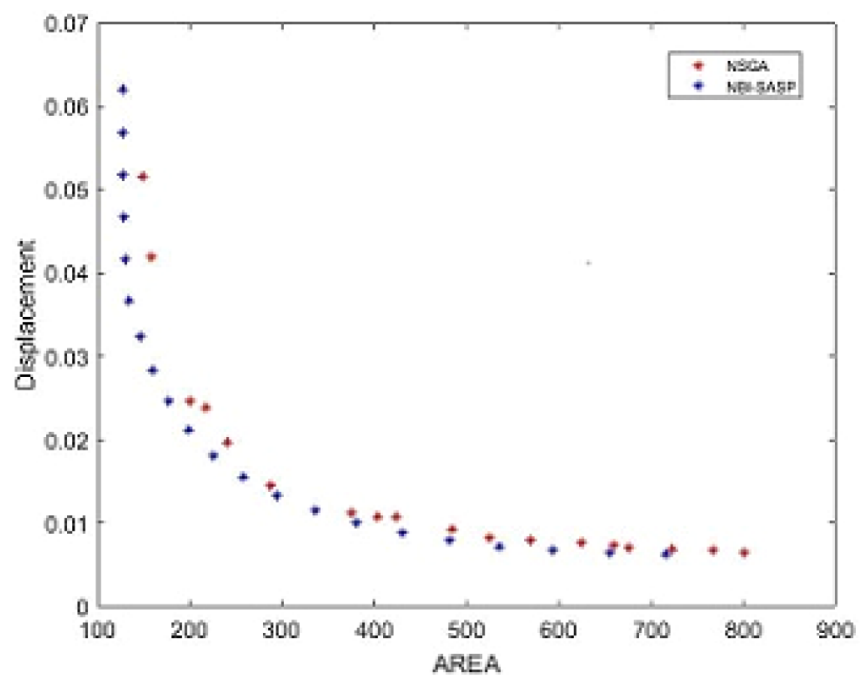

Fig. 9. The solutions obtained by NBI-SASP and NSGA-II on beam design example.

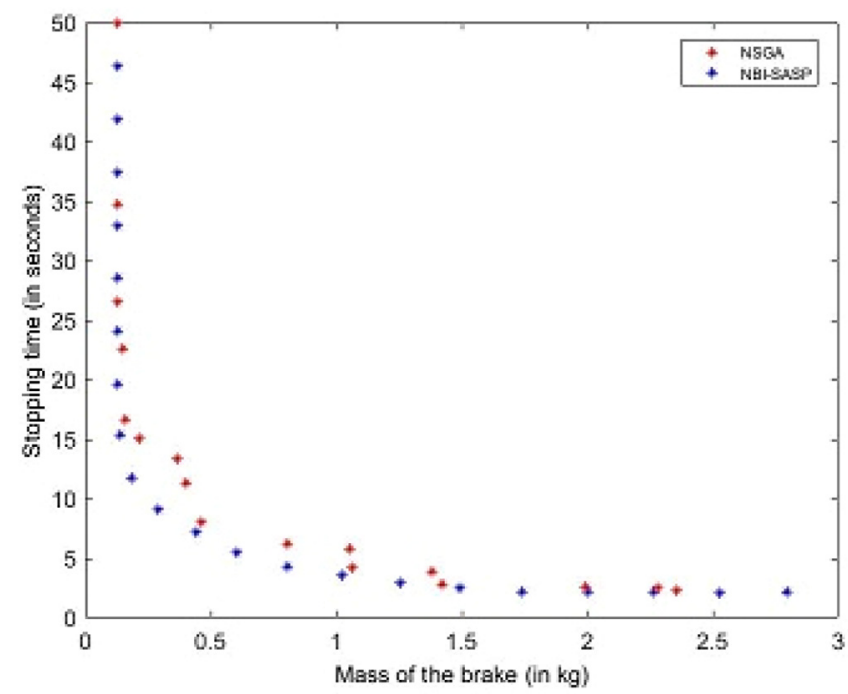

Fig. 10. The solutions obtained by NBI-SASP and NSGA-II on beam design example.

sufficiently show that the NBI SPSA method can come up with efficient, uniformly distributed, near-complete and near optimal Pareto-optimal solutions for multi-objective optimization.

\section{Conclusions}

This study has proposed the Normal Boundary Intersection approach (NBI) based on the SASP method to solve multi-objective design problems. This method is capable of dealing with non-convex objective space, as well as it yields a well distributed Pareto front. The resulting solution given by the suggested approach for the two bar truss design problem, the I-Beam design problem and the disk brake 
design problem is favorable in contrast to the result given by NSGA-II. Furthermore, the aforementioned results propose that the system of optimization suggested is more efficient and practical for solving real-world application problems. For upcoming research, more practical tests shall be done on the algorithm to show its application. Thus, future modifications and improvements of the algorithm are still possible.

\section{References}

1. D. Bassir, F.-X. Irisarri, J.-F. Maire, N. Carrere, Incorporating industrial constraints for multiobjective optimization of composite laminates using a GA, Int. J. Simul. Multidisci. Des. Optim. 2, 101-106 (2008)

2. K. Deb, Current trends in evolutionary multi-objective optimization, Int. J. Simul. Multidisci. Des. Optim. 1, 1-8 (2007)

3. R. El Maani, S. Elouardi, B. Radi, A. El Hami, Multiobjective aerodynamic shape optimization of NACA0012 airfoil based mesh morphing, Int. J. Simul. Multidisci. Des. Optim. 11, $1-10(2020)$

4. A. Tchvagha Zeine, N. El hami, S. Ouhimmou, R. Ellaia, A. Elhami, Multiobjective optimization of trusses using Backtracking Search Algorithm, Incertitudes et fiabilité des systèmes multiphysiques 1, 1-10 (2017)

5. M. Duran Toksari, A heuristic approach to find the global optimum of function, J. Comput. Appl. Math. 209, 160-166 (2007)

6. M. Ehrgott, X. Gandibleux, Multiobjective combinatorial optimization-theory, methodology and applications, in Multiple Criteria Optimization-State of the Art Annotated Bibliographic Surveys, edited by M. Ehrgott and X. Gandibleux. International Series in Operations Research and Management Science (Springer, Boston, MA, 2003), vol 52, pp. 369-444

7. V. Chankong, Y.Y. Haimes, Multiobjective Decision Making Theory and Methodology (North- Holland, New York, 1983)

8. K. Miettinen, Nonlinear Multiobjective Optimization (Kluwer, Boston, 1999)

9. D. Goldberg, Genetic Algorithms in Search, Optimization, and Machine Learning (Addison-Wesley Pub. Co., 1989)

10. C.R. Houck, J. Joines, M. Kay, A genetic algorithm for function optimization: A matlab implementation, Technical Report NCSU-IE-TR-95-09, North Carolina State University, Raleigh, NC, 1995

11. R.E. de Castro, A Genetic Algorithm for Multiobjective Structural Optimization", IV Simposio Mineiro de Mecanica Computacional (2000) 219-226

12. I.G. Tsoulos, Modifications of real code genetic algorithm for global optimization, Appl. Math. Comput. 203, 598-607 (2008)

13. I. Das, J.E. Dennis, Normal boundary intersection, a new methode for generating the pareto surface in nonlinear multicreteria optimization problems, SIAM J. Optim. 3, 631-657 (1998)
14. R. Aboulaich, R. Ellaia, S. El Moumen, The mean-varianceCVaR model for portfolio optimization Modeling using a Multi-Objective Approach based on a hybrid method, Math. Model. Nat. Phenom. 7, 93-98 (2010)

15. S. El Moumen, R. Ellaia, R. Aboulaich, A new hybrid method for solving global optimization problem, Appl. Math. Comput. 218, 3265-3276 (2011)

16. S. Kirpatrick, C.D. Gelatt, M.P. Vecchi, Optimization by simulated annealing, Science 220, 671-680 (1983)

17. N. Metropolis, A.W. Rosenbluth, M.N. Rosenbluth, A.H. Teller, E. Teller, Equations of state calculations by fast computing machines, J. Chem. Phys. 21, 1087-1091 (1953)

18. C.R. Reeves, Modern Heuristic Techniques for Combinatorial Problems (John Wiley and Sons, New York, NY, 1993)

19. J.C. Spall, Multivariate stochastic approximation using a simultaneous perturbation gradient approximation, IEEE Trans. Autom. Control 37, 332-341 (1992)

20. B. Azvine, G.R. Tomlinson, R. Wynne, Use of active constrained-layer damping for controlling resonant vibration, Smart Mater. Struct. 4, 1-6 (1995)

21. M.J. Lam, D.J. Inman, W.R. Saunders, Variations of hybrid damping, in L.P. Davis (ed.), Smart Structures \& Materials 1998: Passive Damping and Isolation, edited by L.P. Davis (SPIE, Bellingham, USA, 1998), Vol. 3327, pp. 32-43

22. S. El Moumen, R. Ellaia, R. Aboulaich, New hybrid algorithm for multi-objective structural optimization, in Proceedings of2013 International Conference on Industrial Engineering and Systems Management (IESM), (2013), pp. 1-5

23. Q. Yuan, Z. He, H. Leng, A hybrid genetic algorithm for a class of global optimization problems with box constraints, Appl. Math. Comput. 197, 924-929 (2008)

24. J. Zhang et al., An effective multiagent evolutionary algorithm integrating a novel roulette inversion operator for engineering optimization, Appl. Math. Comput. 211, 392-416 (2009)

25. J. Schuurmans, J.A. Rossiter, Robust predictive control using tight sets of predicted states, IEE Proc. Control Theory Appl. 147, 13-18 (2000)

26. M. Janga Reddy, D. Nagesh Kumar, An efficient multiobjective optimization algorithm based on swarm intelligence for engineering design, Eng. Optim. 39, 49-68 (2007)

27. K. Deb, Optimal design of a welded beam via genetic algorithms, AIAA J. 29, 2013-2015 (1991)

28. B. Yang, Y. Yeun, W. Ruy, Managing approximation models in multiobjective optimization, Struct Multidiscip Optim. 24, 141-156 (2002)

29. T. Erfani, S.V. Utyuzhnikov, B. Kolo, A modified directed search domain algorithm for multiobjective engineering and design optimization, Struct. Multidiscip. Optim. 48, 1129-1141 (2013)

30. B. Raphael, I.F.C. Smith, A direct stochastic algorithm for global search, Appl. Math. Comput. 146, 729-758 (2003)

31. W. Gong, Z. Cai, L. Zhu, An efficient multiobjective differential evolution algorithm for engineering design, Struct. Multidisc. Optim. 38, 137-157 (2009)

Cite this article as: Samira El Moumen, Siham Ouhimmou New multiobjective optimization algorithm using NBI-SASP approaches for mechanical structural problems, Int. J. Simul. Multidisci. Des. Optim. 13, 4 (2022) 